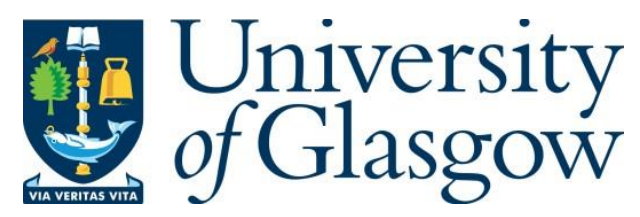

Papamichael, V. and Karadimas, P. (2017) On the covariance matrix and diversity performance evaluation of compact multi-port antenna systems. IEEE Transactions on Antennas and Propagation, (doi:10.1109/TAP.2017.2754439).

There may be differences between this version and the published version. You are advised to consult the publisher's version if you wish to cite from it.

http://eprints.gla.ac.uk/148467/

Deposited on: 20 September 2017

Enlighten - Research publications by members of the University of Glasgow http://eprints.gla.ac.uk 


\section{On the Covariance Matrix and Diversity Performance Evaluation of Compact Multi-Port Antenna Systems}

\author{
Vasilis Papamichael and Petros Karadimas, Member IEEE
}

\begin{abstract}
In this paper, we present a new electromagnetic methodology that determines the covariance matrix of compact multi-port antenna (MPA) systems at the early design stage. Further insight to this field is provided, as contrary to existing methodologies, it rigorously relies on the reciprocity principle of MPA systems. Thus, the impact of propagation environment, termination conditions and MPA radiation characteristics as independent factors affecting performance is physically incorporated. Provided the availability of mathematical expressions for the cumulative distribution function (CDF) and probability density function (PDF) of received signal-to-noise ratio (SNR), performance can be then analytically studied via the diversity antenna gain (DAG) in terms of a) SNR enhancement at a specific outage probability (OP) level (that is DAG-OP) and b) average SNR reduction for achieving a specific average bit error rate (BER) (that is DAG-BER). Illustrative examples with performance evaluation of compact MPA systems are presented. The adopted diversity technique is the transmit antenna selection/maximal ratio combining (TAS/MRC), which for the first time is analytically studied employing realistic MPA systems. Comparisons with existing published results further demonstrate the validity and usefulness of the proposed methodology.
\end{abstract}

Index Terms-Antenna diversity, Covariance matrix methodology, Diversity antenna gain, Multi-port antenna systems.

\section{INTRODUCTION}

WIRELESS communication systems can be greatly improved by the use of antenna diversity techniques. The availability of methods for diversity antenna gain (DAG) evaluation of compact multi-port antenna (MPA) systems at the early design stage is very important.

According to [1], existing methods can be categorized as: a) stochastic electromagnetic simulation methods, b) covariance matrix methods. The benefit of using the covariance matrix, instead of the stochastic electromagnetic simulation, was discussed in [2]. Covariance matrix methods are analytical-based, as they require closed-form cumulative distribution function (CDF) and probability density function (PDF) of the received signal-to-noise-ratio (SNR). They also provide one additional potential for performance evaluation via bit error rate (BER) calculations of the combined signal [3]. The stochastic electromagnetic simulation methods are Monte Carlo-based and apply when no mathematical expressions for the CDF of received SNR are available. In such cases, simulation schemes for modeling the antenna patterns and incoming plane waves are the only option [1]. Alternative non-analytical methods for performance evaluation have employed the use of reverberation chambers emulating rich isotropic multipath environments [4], [5].

In previous approaches, the respective parameters for determining the covariance matrix (i.e., the mean effective gain and correlation coefficient) were affected by the termination conditions, e.g., [2], [6]. The covariance matrix formula in [7] was derived without rigorously applying the reciprocity principle of MPA systems. More specifically, an S-parameter circuit analysis was adopted that included only single polarization of incident fields and led to a covariance matrix formula having an undefined mathematical constant and a factor that ambiguously incorporates antenna radiation characteristics [7, eq. (25)]. That factor lacks of physical electromagnetic interpretation, as for example, the antenna radiation pattern and power gain. The covariance matrix determined in that way did not rigorously comply with the reciprocity principle of MPA systems. The interested reader is referred to [8] regarding the rigorous applicability of the reciprocity principle on MPA systems.

In this paper, we present a new covariance matrix formula by adopting an electromagnetic methodology based on the effective length matrix (eq. (13)). The latter was recently introduced in [1] as a condensed parameter for accurate modeling of the radiation fields of any MPA type by rigorously applying the reciprocity principle in such systems and generalizing the effective length concept of single antennas. Thus, the proposed formula physically incorporates the impact of propagation environment, termination conditions and MPA radiation characteristics on diversity performance evaluation. Both vertical and horizontal polarization of incident fields are further included. Each of the aforementioned factors affecting the performance of MPA systems can be independently studied and compensated at the early design stage. The proposed formula is currently the most complete seen in published literature without undefined quantities. Thus, to further strengthen the necessity of adopting the proposed covariance matrix, we demonstrate the discrepancy appeared in the formula of [7], together with certain conditions under which it appears to be a special case of the proposed one.

Then, based on the new covariance matrix formula and existing mathematical expressions for the CDF and PDF of received SNR, we analytically determine the DAG-OP and DAG-BER (see Abstract for definitions) of compact MPA systems. We employ the transmit antenna selection/maximal ratio combining (TAS/MRC) as a hybrid technique exploiting diversity at both link ends and incorporating MRC as special case [9], [10]. Comparisons with the simulated results from [9] are also presented, thus further confirming the validity and usefulness of the proposed methodology. In [9], the DAG-OP of compact MPA systems under TAS/MRC was just studied using a stochastic electromagnetic simulation method. Complete TAS/MRC performance in terms of DAG-OP and DAG-BER is analytically studied for the first time in this paper, when incorporating termination conditions and radiation characteristics of realistic MPA systems. 
Although the focus of this paper is on diversity performance of MPA systems, the proposed electromagnetic methodology can be advantageous to other research fields requiring covariance matrix estimations, such as MIMO capacity calculations [11]-[13] and antenna systems for direction of arrival (DOA) estimation [14], [15]. In both fields, the covariance matrix was not determined via the rigorous applicability of the reciprocity principle of MPA systems [8]. Thus, they did not physically account for the termination conditions and MPA radiation characteristics via metrics complying with standardized IEEE terminology [16]. As a result, such covariance matrix estimations did not explicitly incorporate the termination circuit's characteristics, e.g., compare the proposed formula in eq. (13) with [12, eq. (10)] and [15, eq. (12)]. Moreover, in MIMO capacity calculations, correlation matrix estimations of the end-to-end channel matrix are required, e.g., see [11, eqs. (5), (6)]. The correlation matrix can degenerate to a Kronecker product of covariance matrices at the $\mathrm{Tx}$ and $\mathrm{Rx}$ sides under certain conditions, e.g., see [12, eq. (18)], [13, eq. (16)]. Accordingly, optimization of diversity performance of MPA systems can potentially lead to MIMO capacity maximization by accurately estimating such covariance matrices via the proposed methodology [17], [18].

The remainder of this paper is organized as follows: Section II presents the proposed covariance matrix formula and a mathematical analysis demonstrating the discrepancy of [7, eq. (25)]. Section III derives the DAG-OP and DAG-BER under TAS/MRC diversity. Results and comparisons with previously published works are shown in Section IV. Finally, Section V draws the conclusion.

\section{Covariance Matrix Methodology}

The covariance matrix has been determined using the mean effective gain and correlation coefficient of the antenna elements [2], [6], or using the non-rigorous formula [7, eq. (25)] having an undefined mathematical constant and a quantity that ambiguously incorporates antenna radiation characteristics. First, we mathematically demonstrate the discrepancy of [7, eq. (25)] due to the non-rigorous applicability of the reciprocity principle. This further necessitates the adoption of the proposed methodology that accurately determines the covariance matrix decomposing the impact of termination conditions, MPA radiation characteristics and propagation environment.

\section{A. The Covariance Matrix Formula [7, eq. (25)]}

Comparing [1, eq. (13)] and [7, eq. (23)] and considering single polarization as in [7] (e.g., vertical polarization), we have after some manipulations

$$
\ell_{\vartheta, \mathrm{m}}(\Omega)=2 \mathrm{c}_{1} \mathrm{~F}_{\vartheta, \mathrm{m}}(\Omega)
$$

where $\Omega$ is the direction in solid angle, $\ell_{\vartheta, m}(\Omega)$ the effective length of element $m$ in vertical polarization when the others are open-circuited [1] and $\mathrm{c}_{1}$ the undefined mathematical constant from [7]. In [7, eq. (21)], $F_{\vartheta, m}(\Omega)$ is defined as the vector far field radiation pattern of the element $\mathrm{m}$ when the others are open-circuited. However, the way it is defined cannot justify the use of the terminology "radiation pattern" as it is an auxiliary undetermined factor used to justify the dependency of the far field radiation pattern on how the antenna ports are fed, see also [8, eq. (15)]. In fact, $F_{\vartheta, m}(\Omega)$ ambiguously incorporates directional radiation characteristics without a physical electromagnetic interpretation, as for example, the antenna radiation pattern and power gain do. Such conclusion is deduced by comparing the similar equations $[8$, eq. (15)] and [7, eq. (21)] with the latter being just the normalized version of the former. Accordingly, $\mathrm{c}_{1}$ is not just a scaling constant, but it has an ambiguous dependency on the way the ports are fed and the directional characteristics of the MPA system.

We further validate the aforementioned conclusion by determining the MPA radiation pattern $\mathrm{E}(\Omega)$ as

$\mathrm{E}(\Omega)=\mathrm{E}_{\mathrm{T}, \vartheta}(\mathrm{r}, \Omega) / \max _{(\Omega)}\left[\mathrm{E}_{\mathrm{T}, \vartheta}(\mathrm{r}, \Omega)\right]$

where $\mathrm{E}_{\mathrm{T}, 9}(\mathrm{r}, \Omega)$ is the vertically polarized radiated electric field of the MPA and $\max _{(\Omega)}[$.] the maximum value of the term in bracket with respect to $\Omega$. Using [1, eq. (8)], we obtain

$$
\mathrm{E}(\Omega)=\frac{\sum_{\mathrm{m}} \ell_{\vartheta, \mathrm{m}}(\Omega) \mathrm{I}_{\mathrm{m}}}{\max _{(\Omega)}\left[\sum_{\mathrm{m}} \ell_{\vartheta, \mathrm{m}}(\Omega) \mathrm{I}_{\mathrm{m}}\right]}
$$

where $I_{m}$ is the excitation current of element $m$ when the others are open-circuited. Comparing (3) and [7, eq. (21)] and taking into account that both formulas arise by employing the superposition principle, we take after some manipulations

$\ell_{\vartheta, \mathrm{m}}(\Omega)=\max _{(\Omega)}\left[\sum_{\mathrm{m}} \ell_{\vartheta, \mathrm{m}}(\Omega) \mathrm{I}_{\mathrm{m}}\right] \times \mathrm{F}_{\vartheta, \mathrm{m}}(\Omega)$

Comparing (1) and (4) we have

$2 \mathrm{c}_{1}=\max _{(\Omega)}\left[\sum_{\mathrm{m}} \ell_{\vartheta, \mathrm{m}}(\Omega) \mathrm{I}_{\mathrm{m}}\right]$

Eq. (5) validates that $c_{1}$ depends on the specific direction in space (one specific direction among infinite choices) that maximizes the sum at the right side of (5), with the excitation currents to be defined accordingly. In fact, $\mathrm{c}_{1}$ appears to be 
determined when excitation currents $I_{m}$ align in phase the complex quantities $\ell_{\vartheta, \mathrm{m}}(\Omega)$, whereas their amplitudes are proportional to the amplitudes of $\ell_{\vartheta, \mathrm{m}}(\Omega)$. The latter resembles to the MRC setting, e.g., [19, p. (852)]. Thus, the solution to (5) will be of the form

$$
\begin{aligned}
\mathrm{c}_{1}=\mathrm{A} \times \max _{(\Omega)}\left[\sum_{\mathrm{m}}\left|\ell_{\vartheta, \mathrm{m}}(\Omega)\right|^{2}\right] \stackrel{[1, \mathrm{eq} \cdot(6)]}{=} \\
\mathrm{B} \times \max _{(\Omega)}\left[\sum_{\mathrm{m}} \operatorname{Re}\left(\mathrm{Z}_{\mathrm{mm}}\right) \mathrm{G}_{\vartheta, \mathrm{m}}(\Omega)\right]
\end{aligned}
$$

where $Z_{m m}$ is the input impedance and $G_{\vartheta, m}(\Omega)$ the power gain pattern of element $\mathrm{m}$ in vertical polarization. $\mathrm{A}$ and $\mathrm{B}$ are proportionality factors accounting for the constant terms (independent of $\Omega$ ) of the formula [1, eq. (6)] relating $\ell_{\vartheta, \mathrm{m}}(\Omega)$ and $\mathrm{G}_{\vartheta, \mathrm{m}}(\Omega) . \mathrm{G}_{\vartheta, \mathrm{m}}(\Omega)$ is determined by [1, eq. (4)] employing $Z_{m m}, I_{m}$, and $f_{\vartheta, m}(\Omega)$, with $f_{\vartheta, m}(\Omega)$ being the $\vartheta$ component of the radiation vector $f_{m}(\Omega)$ of element $\mathrm{m} . \mathrm{f}_{\mathrm{m}}(\Omega)$ is determined by the MPA's current density when exiting element $\mathrm{m}$ and having the others open-circuited and is mathematically defined in [20, eq. (15.7.5)]. $\mathrm{G}_{\varphi, \mathrm{m}}(\Omega)$, i.e., the power gain pattern of element $\mathrm{m}$ in horizontal polarization, is similarly determined by employing the $\varphi$ component $f_{\varphi, m}(\Omega)$ of $f_{m}(\Omega)$.

Thus, $\mathrm{c}_{1}$ depends on the spatial direction that the sums in (6) are maximized and its determination requires numerical techniques. No analytical generic solution can thus be found, as it will rely on the specific MPA setting and application of suitable numerical techniques. This discrepancy is directly transferred to the covariance matrix determination in [7, eq. (25)]. Thus, the performance of realistic MPA systems cannot physically/electromagnetically rely on such a formula employing $\mathrm{c}_{1}$. The reason is the non rigorous applicability of the reciprocity principle in [7, eq. (23)], as is theoretically well-known and valid in MPA systems [8]. In the next subsection, we will overcome this discrepancy by adopting an electromagnetic methodology that rigorously complies with the reciprocity principle of MPA systems.

\section{B. Proposed Methodology}

We begin with the $N x l$ voltage vector $\mathbf{v}_{\mathrm{L}}(\Omega)$ on the MPA's terminal ports, which is excited by an external incident electric field $\mathbf{E}_{\mathrm{R}}(\Omega)$. Thus [1, eq. (12)]

$$
\mathbf{v}_{\mathrm{L}}(\Omega)=\underbrace{\left(\mathbf{I}+\mathbf{Z}^{\mathrm{T}} \mathbf{Z}_{\mathrm{L}}^{-1}\right)^{-1}}_{\mathbf{W}} \mathbf{L}_{\mathrm{e}}(\Omega) \mathbf{E}_{\mathrm{R}}(\Omega)
$$

where $\mathbf{Z}$ is the $N x N$ impedance matrix of the MPA, $\mathbf{Z}_{\mathrm{L}}$ the $N x N$ termination circuit's impedance matrix and $\mathbf{L}_{\mathrm{e}}(\Omega)$ the $N x 2$ effective length matrix [1, eqs. (6), (9)]. Considering equal noise powers at the reference antenna and each port of the MPA, the elements $\Lambda_{\mathrm{ij}}$ of the covariance matrix $\Lambda$ are defined as [21, eq. (10.57)]

$$
\Lambda_{\mathrm{ij}}=\Gamma_{0} \frac{\left\langle\mathrm{v}_{\mathrm{Li}}(\Omega) \mathrm{v}_{\mathrm{L} j}{ }^{*}(\Omega)\right\rangle}{\left\langle\left|\mathrm{v}_{\text {ref }}(\Omega)\right|^{2}\right\rangle}
$$

where $\Gamma_{0}$ and $\mathrm{v}_{\text {ref }}(\Omega)$ are the average signal-to-noise ratio (SNR) and voltage received by the reference antenna, respectively. Using (7) in (8), we express $\boldsymbol{\Lambda}$ after some manipulations as

$\boldsymbol{\Lambda}=\Gamma_{0} \frac{\mathbf{W}\left(\int_{\Omega} \mathbf{L}_{\mathrm{e}}(\Omega) \mathbf{P}^{\prime}(\Omega) \mathbf{L}_{\mathrm{e}}{ }^{\mathrm{H}}(\Omega) \mathrm{d} \Omega\right) \mathbf{W}^{\mathrm{H}}}{\left\langle\left|\mathrm{v}_{\text {ref }}(\Omega)\right|^{2}\right\rangle}$

where the $2 \times 2$ matrix $\mathbf{P}^{\prime}(\Omega)$ accounting for the propagation environment characteristics is defined as

$\mathbf{P}^{\prime}(\Omega)=\left[\begin{array}{cc}\underbrace{\mathrm{C}_{9} \mathrm{p}_{9}(\Omega)}_{\left|\mathrm{E}_{\mathrm{R} \vartheta}(\Omega)\right|^{2}} & 0 \\ 0 & \underbrace{\mathrm{C}_{\varphi} \mathrm{p}_{\varphi}(\Omega)}_{\left|\mathrm{E}_{\mathrm{R} \varphi}(\Omega)\right|^{2}}\end{array}\right]$

where the ratio $\mathrm{C}_{\vartheta} / \mathrm{C}_{\varphi}=\mathrm{XPR}$ is the cross polarization power ratio and $\mathrm{p}_{\vartheta}(\Omega)$ and $\mathrm{p}_{\varphi}(\Omega)$ are the angular power distributions of the vertically and horizontally polarized incident waves, respectively [22]. Using (7), the denominator of (9) is calculated as

$$
\left\langle\left|\mathrm{v}_{\mathrm{ref}}\right|^{2}\right\rangle=\frac{\int_{\Omega}\left[\left|\ell_{\vartheta}^{\mathrm{ref}}\right|^{2} \mathrm{C}_{\vartheta} \mathrm{p}_{\vartheta}(\Omega)+\left|\ell_{\varphi}^{\mathrm{ref}}\right|^{2} \mathrm{C}_{\varphi} \mathrm{p}_{\varphi}(\Omega)\right] \mathrm{d} \Omega}{\left[1+\left(\mathrm{Z}_{\mathrm{ref}} / \mathrm{Z}_{0}\right)\right]^{2}}
$$

where, for the reference antenna, $Z_{\text {ref }}=Z_{0}$ is the input impedance, $Z_{0}$ the transmission line characteristic impedance

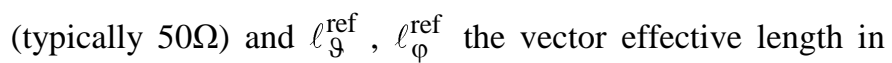
the vertical and horizontal polarizations, respectively. For a dual isotropic reference antenna, they are determined as [1]

$\ell_{\vartheta}^{\mathrm{ref}}=\ell_{\varphi}^{\mathrm{ref}}=\sqrt{\frac{4 \pi \mathrm{Z}_{0}}{\omega \mu_{0} \mathrm{k}}}$ 
where $\omega$ is the angular frequency, $\mu_{0}$ the free space magnetic permeability and $\mathrm{k}$ the wave number. Thus, by combining (9), (11) and (12) with $Z_{\text {ref }}=Z_{0}$, we obtain after some mathematical manipulations

$$
\boldsymbol{\Lambda}=\Gamma_{0} \frac{\omega \mu_{0} \mathrm{k}}{\pi \mathrm{Z}_{0}} \mathbf{W}\left(\int_{\Omega} \mathbf{L}_{\mathrm{e}}(\Omega) \mathbf{P}(\Omega) \mathbf{L}_{\mathrm{e}}{ }^{\mathrm{H}}(\Omega) \mathrm{d} \Omega\right) \mathbf{W}^{\mathrm{H}}
$$

where

$$
\mathbf{P}(\Omega)=\left[\begin{array}{cc}
\frac{\mathrm{XPR}}{\mathrm{XPR}+1} \mathrm{p}_{\vartheta}(\Omega) & 0 \\
0 & \frac{1}{\mathrm{XPR}+1} \mathrm{p}_{\varphi}(\Omega)
\end{array}\right]
$$

Hence, the covariance matrix $\boldsymbol{\Lambda}$ can be accurately calculated for any termination condition, MPA radiation characteristics and propagation environment, employing parameters defined according to standardized IEEE terminology [16]. If single polarization is considered, (13) will have similar form with the covariance matrix formula of [7], when conditioned on (1) with $\mathrm{c}_{1}$ to be defined as in (6). The covariance matrix in (13) needs to be determined at the initial stage employing the effective length matrix $\mathbf{L}_{\mathrm{e}}(\Omega)$ that physically arises from the reciprocity principle of MPA systems. The interested reader is further referred to [1, eqs. (10)-(13)] on how $\mathbf{L}_{\mathrm{e}}(\Omega)$ arises by rigorously applying the reciprocity principle of MPA systems, as demonstrated in [8].

\section{DAG-OP AND DAG-BER UNDER TAS/MRC}

The DAG is determined in terms of SNR enhancement at a specific outage probability (OP) $\mathrm{u}_{1}$ (DAG-OP) and in terms of average SNR reduction for achieving a specific average bit error rate (BER) $\mathrm{u}_{2}$ (DAG-BER) [6]. In order to find both DAGs, the PDF and CDF of received SNR are needed. Considering TAS/MRC diversity in a Rayleigh channel, with $M$ Tx and $N \mathrm{Rx}$ antennas, they are defined as [23]

$$
\begin{aligned}
& \operatorname{PDF}(\mathrm{x})=\mathrm{M}\left[\sum_{\mathrm{i}=1}^{\mathrm{N}} \zeta_{\mathrm{i}}\left(1-\mathrm{e}^{-\mathrm{x} / \lambda_{\mathrm{i}}}\right)\right]^{\mathrm{M}-1} \sum_{\mathrm{i}=1}^{\mathrm{N}} \zeta_{\mathrm{i}} \frac{\mathrm{e}^{-\mathrm{x} / \lambda_{\mathrm{i}}}}{\lambda_{\mathrm{i}}} \\
& \operatorname{CDF}(\mathrm{x})=\int_{0}^{\mathrm{x}} \mathrm{M}\left[\sum_{\mathrm{i}=1}^{\mathrm{N}} \zeta_{\mathrm{i}}\left(1-\mathrm{e}^{-\mathrm{s} / \lambda_{\mathrm{i}}}\right)\right]^{\mathrm{M}-1} \sum_{\mathrm{i}=1}^{\mathrm{N}} \zeta_{\mathrm{i}} \frac{\mathrm{e}^{-\mathrm{s} / \lambda_{\mathrm{i}}}}{\lambda_{\mathrm{i}}} \mathrm{ds}
\end{aligned}
$$

where $\lambda_{i}$ are the eigenvalues of $\Lambda$ and $\zeta_{i}=\prod_{j=1, j \neq i}^{N}\left[\lambda_{i} /\left(\lambda_{i}-\lambda_{j}\right)\right]$.

For the DAG-OP we have [6]
TABLE I

\begin{tabular}{|c|c|c|c|c|c|}
\hline \multicolumn{7}{|c|}{ DAG-OP RESULTS OVERVIEW } \\
\hline TAS/ & TAS/ & TAS/ & TAS/ & MRC-4 & MRC-2 \\
MRC-4 & MRC-4 & MRC-4 & MRC-2 & $Z_{0}$ Term & $Z_{0}$ Term \\
Hermitian & $Z_{0}$ Term. & $Z_{0}$ Term. & $Z_{0}$ Term & {$[24]$} & {$[24]$} \\
Term. & & Vert. Pol. & & & \\
\hline $21 \mathrm{~dB}$ & $17.6 \mathrm{~dB}$ & $12.4 \mathrm{~dB}$ & $13.5 \mathrm{~dB}$ & $14.1 \mathrm{~dB}$ & $8 \mathrm{~dB}$ \\
\hline
\end{tabular}

TABLE II

DAG-BER RESULTS OVERVIEW

\begin{tabular}{|c|c|c|c|c|c|}
\hline TAS/ & TAS/ & TAS/ & TAS/ & MRC-4 & MRC-2 \\
MRC-4 & MRC-4 & MRC-4 & MRC-2 & $Z_{0}$ Term & $Z_{0}$ Term \\
Hermitian & $Z_{0}$ Term. & $\begin{array}{c}Z_{0} \text { Term. } \\
\text { Term. }\end{array}$ & $Z_{0}$ Term & {$[6]$} & {$[6]$} \\
\hline $30.6 \mathrm{~dB}$ & $27.2 \mathrm{~dB}$ & $22 \mathrm{~dB}$ & $22.7 \mathrm{~dB}$ & $23.5 \mathrm{~dB}$ & $14.8 \mathrm{~dB}$ \\
\hline
\end{tabular}
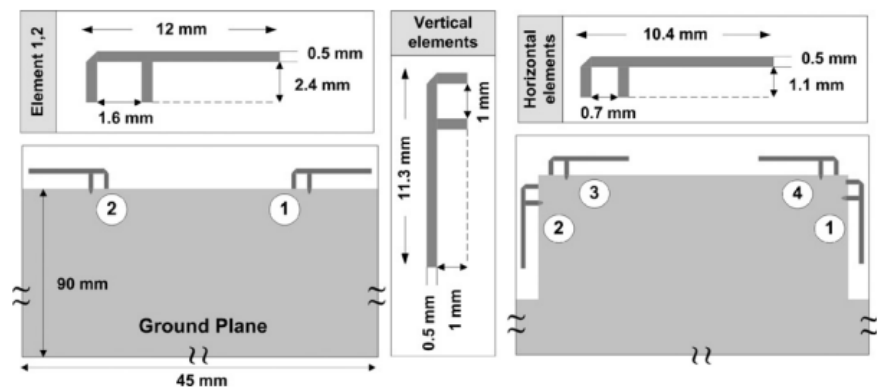

Fig.1. Layout of the investigated MPA systems [1].

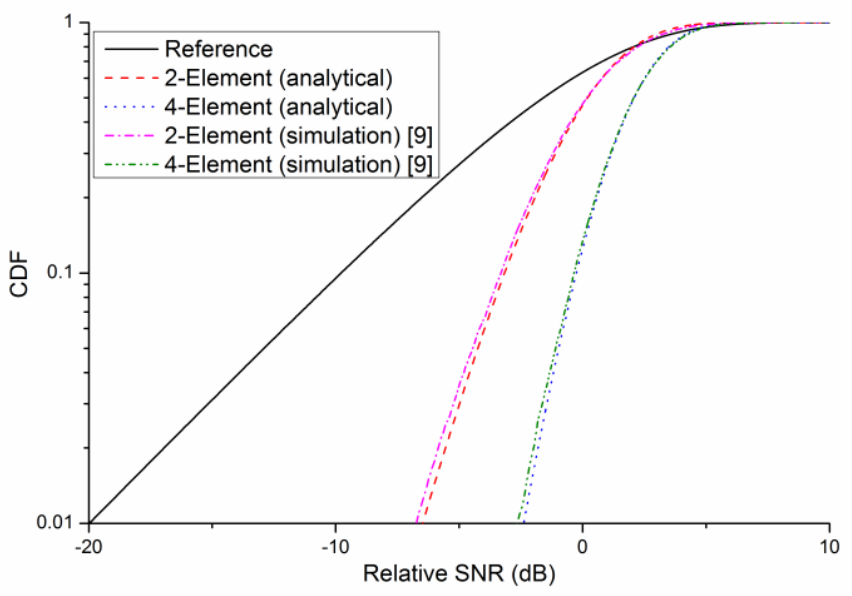

Fig.2. CDF of relative SNR for the reference antenna and the 2- and 4-element MPAs under TAS/MRC.

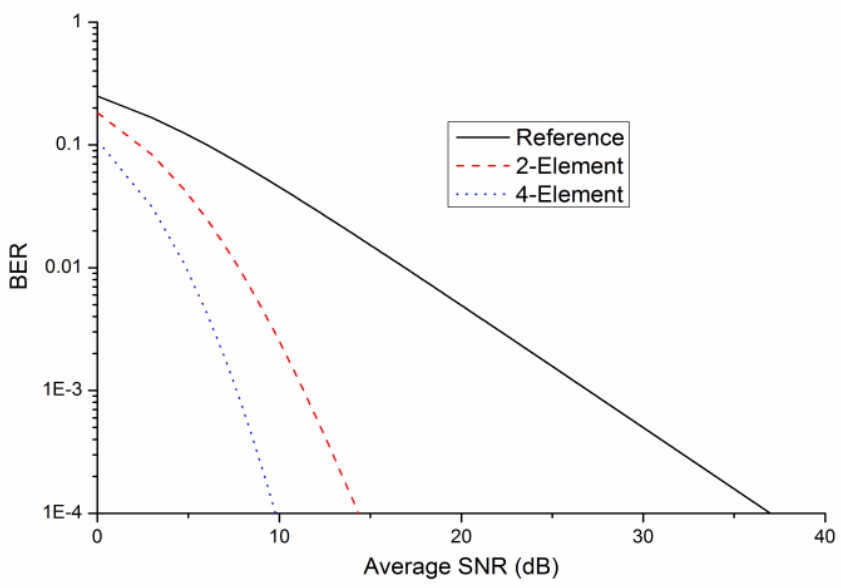

Fig.3. BER of DPSK signalling for the reference antenna and the 2- and 4-element MPAs under TAS/MRC. 


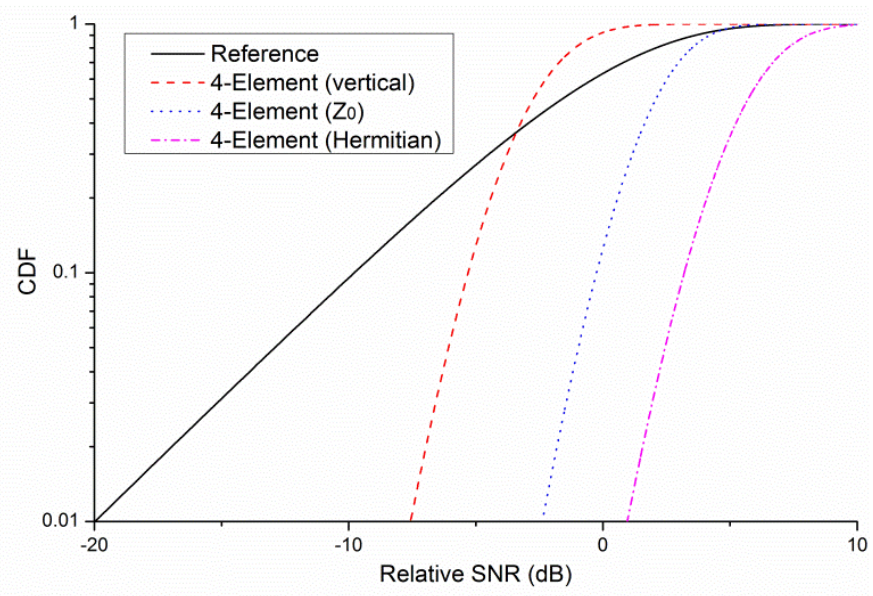

Fig.4. Case studies for CDF of relative SNR under TAS/MRC.

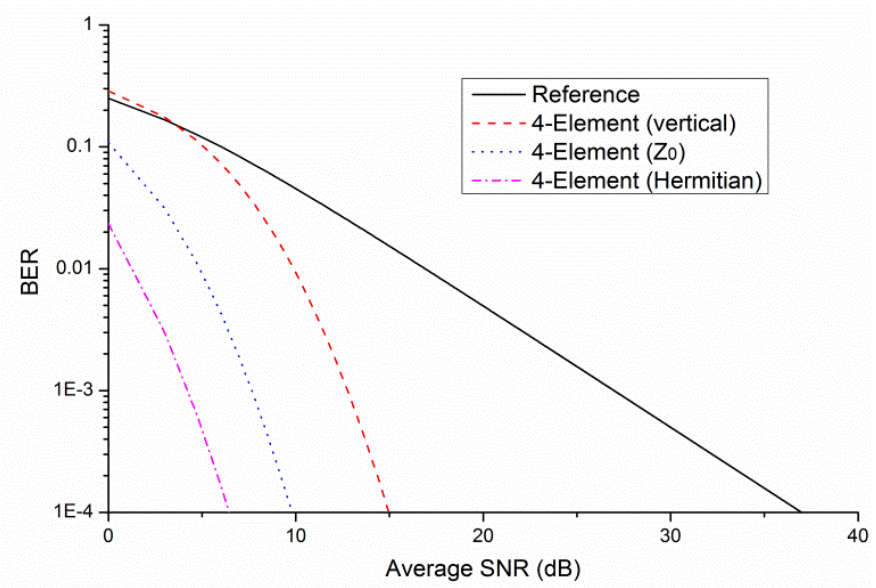

Fig.5. Case studies for BER of DPSK signalling under TAS/MRC.

$$
\mathrm{DAG}-\mathrm{OP}=\left.\frac{\mathrm{x}_{1}}{\mathrm{x}_{2}}\right|_{\mathrm{P}\left(\gamma_{\mathrm{D}} \leq \mathrm{x}_{1}\right)=\mathrm{P}\left(\gamma_{0} \leq \mathrm{x}_{2}\right)=\mathrm{u}_{1}}
$$

where $\quad \mathrm{P}\left(\gamma_{\mathrm{D}} \leq \mathrm{x}_{1}\right)=\mathrm{CDF}\left(\mathrm{x}_{1}\right) \quad$ and $\quad \mathrm{P}\left(\gamma_{0} \leq \mathrm{x}_{2}\right)=$ $1-\exp \left(-\mathrm{x}_{2} / \Gamma_{0}\right)$ are derived from the CDFs of received SNR of the MPA and reference antenna, respectively. The DAG-BER is defined as [3], [6]

$$
\mathrm{DAG}-\mathrm{BER}=\left.\frac{\Gamma_{0}}{\Gamma_{\mathrm{D}}}\right|_{\mathrm{P}_{\mathrm{e}}\left(\Gamma_{\mathrm{D}}\right)=\mathrm{P}_{\mathrm{e}}\left(\Gamma_{0}\right)=\mathrm{u}_{2}}
$$

where $\Gamma_{\mathrm{D}}$ and $\Gamma_{0}$ are the average SNRs of the MPA and reference antenna, respectively. Considering DPSK signaling, we obtain the BER of the MPA and reference antenna as $\mathrm{P}_{\mathrm{e}}\left(\Gamma_{\mathrm{D}}\right)=\int_{0}^{\infty}[\operatorname{PDF}(\mathrm{x}) \exp (-\mathrm{x}) / 2] \mathrm{dx} \quad$ and $\quad \mathrm{P}_{\mathrm{e}}\left(\Gamma_{0}\right)=$ $1 /\left(2+2 \Gamma_{0}\right)[21$, p. (293)], respectively.

\section{RESULTS}

The geometry and dimensions of the investigated MPA configurations are depicted in Fig.1 [1]. They have typical dimensions of a PC card, whereas the dimensions of the ground plane are $45 \mathrm{~mm}$ by $90 \mathrm{~mm}$. The investigated MPA systems comprise of 2 and 4 inverted-F antenna elements operating in the 5.2 GHz ISM band. For more details see [24]. In order to calculate the diversity performance of these systems and make fair comparisons with existing published results, we determine the covariance matrix assuming a uniform propagation environment as in [6] and [9], i.e., $\mathrm{XPR}=1$ and $\mathrm{p}_{\vartheta}(\Omega)=\mathrm{p}_{\varphi}(\Omega)=1 /(4 \pi)$. In Figs.2 and 3 , the termination load impedance matrix is $\mathbf{Z}_{\mathrm{L}}=\mathrm{Z}_{0} \mathbf{I}$. We further assume two Tx antennas as in [9]. Results are summarized in Tables I, II and discussed in the following.

Initially, the DAG-OP at $1 \%$ OP is calculated $\left(\mathrm{u}_{1}=0.01\right)$. The CDFs of received SNR of the two MPAs and reference antenna are shown for that purpose in Fig.2. The SNR has been normalized to the average SNR of the reference antenna (i.e., $\left.\Gamma_{0}=1\right)$. The calculated DAG-OPs at $1 \%$ OP are 13.5 and 17.6 $\mathrm{dB}$ for the 2- and 4-element antennas, respectively. Comparisons with the simulated results published in [9] are also shown, where an excellent conformity is revealed. The respective DAG-OPs were 13.2 and $17.3 \mathrm{~dB}$.

The DAG-BER for achieving a $10^{-4}$ BER is then calculated $\left(\mathrm{u}_{2}=0.0001\right)$. Thus in Fig.3, we present the calculated BERs of the two MPAs and reference antenna with respect to the average SNR. The DAG-BERs are 22.7 and $27.2 \mathrm{~dB}$ for the 2and 4-element antenna, respectively. In [6], the same MPAs were tested in similar propagation conditions, operating under MRC. The respective DAG-BERs were 14.8 and $23.5 \mathrm{~dB}$ employing the same signalling scheme. Thus, TAS/MRC, when compared to MRC, shows a profound improvement in terms of BER performance, penalized through a slight increase in complexity (i.e., employing just two Tx antennas).

In Figs.4 and 5, we demonstrate that performance can be heavily underestimated when single polarization is considered, whereas it can be potentially improved by altering the termination conditions. The DAG-OP $(1 \% \mathrm{OP})$ and DAG-BER $\left(10^{-4} \mathrm{BER}\right)$ for the case of vertical polarization only are 12.4 and $22 \mathrm{~dB}$, respectively, i.e., $5.2 \mathrm{~dB}$ less compared to the case of including both polarizations. Moreover, Hermitian match termination increases both DAG-OP (1\% OP) and DAG-BER $\left(10^{-4} \mathrm{BER}\right)$ performance by approximately $3.4 \mathrm{~dB}$, as in such case, these are 21 and $30.6 \mathrm{~dB}$, respectively.

The 4-element antenna with vertical polarization performs slightly worse compared to the reference in the low average SNR regions, i.e., at about less than $5 \mathrm{~dB}$ in Fig.5. This is because single polarization neglects almost $50 \%$ of total received power, whereas the elements have an efficiency of about $60 \%$ [24]. We also notice from Figs.3 and 5 that performance improvement becomes more evident at the higher average SNR regimes when employing TAS/MRC antenna diversity. On the contrary, the lower average SNR regions do not show such performance improvement. Similar trends have been observed when employing other diversity techniques such as selection combining (SC) [2] and MRC [3], [6]. 


\section{CONCLUSION}

A new accurate expression for the covariance matrix of MPA systems has been presented by adopting a physical electromagnetic methodology. As opposed to existing ones, the proposed methodology rigorously complies with the reciprocity principle of MPA systems via the vector effective length matrix. Thus, propagation environment, termination conditions and MPA radiation characteristics are accounted as independent factors affecting performance via metrics complying with standardized IEEE terminology and without using undefined or ambiguous quantities. We have then analytically derived BER-based and OP-based performance of MPA systems operating under TAS/MRC diversity. Excellent conformity with published simulated results has been revealed. Significant BER-based performance improvement compared to MRC has been shown. Considering single polarization can heavily underestimate performance, whereas altering termination conditions can potentially improve performance.

The proposed covariance matrix electromagnetic methodology is extremely important, because it does not rely on propagation channel measurements and simulations. New research can be further undertaken by applying this methodology to other well-known channel models representing physical reality (e.g., clustered-based Nakagami, LOS Rician), and alternative diversity techniques (e.g., generalized selection combining). The only requirement is the availability of mathematical expressions for the CDF and PDF of received SNR. It is thus deduced that the proposed physical electromagnetic modeling of the covariance matrix provides new insight, constituting a powerful tool for performance evaluation and comparison of different MPA systems at the early design stage, operating under any diversity technique, propagation environment and termination conditions. As covariance matrix estimation is the core for other research fields, such as MIMO capacity calculations and antenna systems for DOA estimation, the proposed methodology can potentially benefit and lead to new research directions in those fields too.

\section{REFERENCES}

[1] V. Papamichael and C. Soras, "Generalized Selection Combining Diversity Performance of Multi-Element Antenna Systems via a Stochastic Electromagnetic-Circuit Model," IET Microwaves, Antennas and Propagation, vol. 4, no. 7, pp. 837-846, Jul. 2010.

[2] V. Papamichael, "Selection-Combining Diversity Performance of actual Multielement Antenna Systems Using the Covariance Matrix Method," IEEE Antennas and Wireless Propagation Letters, vol. 9, pp. 705-707, 2010.

[3] K. Ogawa and J. Takada, "An Analysis of the Effective Performance of a Handset Diversity Antenna Influenced by Head, Hand, and Shoulder Effects-A Proposal for a Diversity Antenna Gain Based on a Signal Bit Error Rate and Analytical Results for the PDC System," Electronics and Communications in Japan, Part 2, vol. 84, no. 6, pp. 10-23, Jun. 2001.

[4] X. Chen, P-S. Kildal, J. Carlsson and J. Yang, "MRC Diversity and MIMO Capacity Evaluations of Multi-Port Antennas Using Reverberation Chamber and Anechoic Chamber," IEEE Transactions on Antennas and Propagation, vol. 61, no. 2, pp. 917-926, Feb. 2013.

[5] A. Hussain, A. A. Glazunov, B. P. Einarsson and P-S. Kildal, "Antenna Measurements in Reverberation Chamber using USRP," IEEE Transactions on Antennas and Propagation, vol. 64, no. 3, pp. 1152-1157, Mar. 2016.
[6] V. Papamichael, M. Karaboikis, C. Soras and V. Makios, "Diversity and MIMO Performance Evaluation of Common Phase Center Multi Element Antenna Systems," Radioengineering, vol. 17, no. 2, pp. 32 - 36, Jun. 2008.

[7] J. W. Wallace and M. A. Jensen, "Termination-Dependent Diversity Performance of Coupled Antennas: Network Theory and Analysis,"IEEE Transactions on Antennas and Propagation, vol. 52, no. 1, pp. 98-105, Jan. 2004.

[8] A. T. De Hoop, 'The N-port Receiving Antenna and its Equivalent Electrical Network," Philips Res. Repts., vol. 30, pp. 302-315, 1975.

[9] V. C. Papamichael and P. Karadimas, "Performance Evaluation of Actual Multielement Antenna Systems Under Transmit Antenna Selection/Maximal Ratio Combining," IEEE Antennas and Wireless Propagation Letters, vol.10, pp. 690-692, 2011.

[10] S. Thoen, L. Van der Perre, B. Gyselinckx and M. Engels, "Performance Analysis of Combined Transmit-SC/Receive-MRC," IEEE Transactions on Communications, vol. 49, no. 1, pp. 5-8, Jan. 2001.

[11] J. O. Nielsen, B. Yanakiev, S. C. Del Bario and G. F. Pedersen, "Channel Models for Capacity Evaluation of MIMO Handsets in Data Mode," IET Microwaves, Antennas and Propagation, vol. 11, no. 1, pp. 1-9, Jan. 2017.

[12] S. K. Yong and J. S. Thompson, "Three-Dimensional Spatial Fading Correlation Models for Compact MIMO Receivers," IEEE Transactions on Wireless Communications, vol. 4, no. 6, pp. 2856-2869, Nov. 2005.

[13] J. - H. Lee and C. - C. Cheng, "Spatial Correlation of Multiple Antenna Arrays in Wireless Communication Systems," Progress in Electromagnetics Research, vol. 132, pp. 347-368, 2012.

[14] L. C. Godara, "Application of Antenna Arrays to Mobile Communications, Part II: Beam-Forming and Direction-of-Arrival Considerations," Proceedings of the IEEE, vol. 85, no. 8, pp. 1195-1245, Aug 1997.

[15] A. L. Kintz and I. J. Gupta, "A Modified MUSIC Algorithm for Direction of Arrival Estimation in the Presence of Antenna Array Manifold Mismatch," IEEE Transactions on Antennas and Propagation, vol. 64, no. 11, pp. 4836-4847, Nov. 2016.

[16] IEEE Std 145-1993, "IEEE Standard Definitions of Terms for Antennas", pp. 1-32, 1993.

[17] S. Loyka, and A, Kouki, "New Compound Upper Bound on MIMO Channel Capacity," IEEE Communications Letters, vol. 6, no. 3, pp. 96-98, Mar. 2002.

[18] B. T. Quist and M. A. Jensen, "Optimal Antenna Radiation Characteristics for Diversity and MIMO Systems," IEEE Transactions on Antennas and Propagation, vol. 57, no. 11, pp. 3474-3481, Nov. 2009.

[19] J. G. Proakis and M. Salehi, "Digital Communications," $5^{\text {th }}$ ed., McGraw Hill, 2008.

[20] S. J. Orfanidis, "Electromagnetic Waves and Antennas," New Brunswick, NJ: Rutgers University, 2002.

[21] W. C. Y. Lee, "Mobile Communications Engineering - Theory and Applications, $2^{\text {nd }}$ ed." Mc Graw Hill, 1997.

[22] T. Taga, "Analysis for Mean Effective Gain of Mobile Antennas in Land Mobile Radio Environments," IEEE Transactions on Vehicular Technology, vol. 39, no. 2, pp. 117-131, May 1990.

[23] L. Yang, D. Tang and J. Qin, "Performance of Spatially Correlated MIMO Channel with Antenna Selection," Electronics Letters, vol. 40, no. 20, pp. 1281-1282, Sep. 2004.

[24] M. Karaboikis, V. Papamichael, G. Tsachtsiris, C. Soras, and V. Makios, "Integrating Compact Printed Antennas onto Small Diversity/MIMO Terminals," IEEE Transactions on Antennas and Propagation, vol. 56, no. 7, pp. 2067-2078, Jul. 2008. 